\title{
Natural Variation at the Pi-ta Rice Blast Resistance Locus
}

\author{
Yulin Jia, Gregory T. Bryan, Leonard Farrall, and Barbara Valent
}

First author: USDA-ARS, Dale Bumpers National Rice Research Center, Stuttgart, AR 72160-0287; second author: AgResearch Grasslands, Private Bag 11008, Palmerston North, New Zealand; third author: Biological and Chemical Sciences and Engineering, Central Research and Development, P.O. Box 80328, Wilmington, DE 19880-0328; and fourth author: Department of Plant Pathology, Kansas State University, 4024 Throckmorton Plant Science Center, Manhattan, KS 66506-5502.

Accepted for publication 28 July 2003.

\begin{abstract}
Jia, Y., Bryan, G. T., Farrall, L., and Valent, B. 2003. Natural variation at the Pi-ta rice blast resistance locus. Phytopathology 93:1452-1459.

The resistance gene $P i$-ta protects rice crops against the fungal pathogen Magnaporthe grisea expressing the avirulence gene AVR-Pita in a gene-for-gene manner. Pi-ta, originally introgressed into japonica rice from indica origin, was previously isolated by positional cloning. In this study, we report the nucleotide sequence of a 5,113-base pair region containing a japonica susceptibility pi-ta allele, which has overall $99.6 \% \mathrm{nu}-$ cleotide identity to the indica $\mathrm{Pi}$-ta allele conferring resistance. The intron region shows the levels of sequence diversity that typically differentiate

genes from indica and japonica rices, but the other gene regions show less diversity. Sequences of the Pi-ta allele from resistant cultivars Katy and Drew from the southern United States are identical to the resistance $P i$-ta sequence. Sequences from susceptible cultivars El Paso 144 and Cica 9 from Latin America define a third susceptibility haplotype. This brings the total number of $P i$-ta haplotypes identified to four, including the resistance allele and three susceptibility alleles. The Pi-ta locus shows low levels of DNA polymorphism compared with other analyzed $R$ genes. Understanding the natural diversity at the Pi-ta locus is important for designing specific markers for incorporation of this $R$ gene into ricebreeding programs.
\end{abstract}

Rice (Oryza sativa L.) is one of the most important food crops in the world, and the rice blast disease caused by the fungal pathogen Magnaporthe grisea (Hebert) Barr is one of the most damaging diseases of rice (33). Rice blast disease follows a classical gene-for-gene system $(25,29)$, in which a pathogen strain expressing an avirulence $(A V R)$ gene triggers the corresponding resistance $(R)$ gene-mediated defense response. Incorporation of individual $R$ genes into existing rice cultivars has not achieved reliable, longlasting resistance to blast disease because of the high potential for $A V R$ gene variation in the pathogen (29). Pyramiding $R$ genes for achieving effective blast control requires molecular markers for introducing diverse genes into agronomically useful cultivars by marker-assisted selection (MAS). Thus far, more than 20 major blast $R$ genes and numerous quantitative trait loci have been identified, and many of these have been associated with molecular markers $(9,18,24,32)$. The utility of MAS for pyramiding blast $R$ genes has been demonstrated experimentally (9).

Cloning $R$ genes and determining the structural differences that confer resistance or susceptibility phenotypes (functional polymorphisms) provide the ideal molecular markers for introducing these genes into agronomically useful cultivars by MAS. Two of the major blast resistance genes, $P i$ - $t a$ and $P i$ - $b$, have been cloned and characterized at the molecular level $(3,30)$. Both genes encode a predicted cytoplasmic protein with a centrally located nucleotide binding site (NBS) and a carboxyl terminal leucine-rich repeat (LRR) region. $P i-b$ is a member of a small gene family, with tightly linked homologs occurring at a complex locus (30). Pi-ta is located near the centromere on rice chromosome 12 , a region reported to include the closely linked or allelic $P i-t a^{2}$ gene, as well as $P i-4 a(t), P i-4 b(t), P i-6(t)$, and others $(5,20,24,32)$. Cloning of $P i$ - $t a$ identified a simple, single-copy $R$ gene locus with no gene candidates for $P i-t a^{2}$ or the other linked $R$ genes (3). Characteriza-

Corresponding author: B. Valent; E-mail address: bvalent@ksu.edu

Publication no. P-2003-0922-03R

(C) 2003 The American Phytopathological Society tion of the Pi-ta allele in a series of resistant and susceptible cultivars identified a functional polymorphism in the coding sequence; that is, forms of the Pi-ta protein with alanine as amino acid residue 918 are functionally resistant and forms of the pi-ta protein with serine at residue 918 confer susceptibility.

Longer term, understanding the molecular mechanisms underpinning $R$ gene-mediated resistance holds promise for novel strategies for developing durably resistant crops. Rice together with the blast fungus have become a model genetic system for understanding cereal-fungal pathosystems, because both are amenable to genetic analysis and whole genome sequences are publicly available for both rice (31; online, Rice GD of the Beijing Genomics Institute) and the blast fungus (online, Magnaporthe grisea Database of the Whitehead Institute Center for Genome Research). $P i$ - $t a$ is currently unique among cloned cereal $R$ genes in that its corresponding AVR gene, AVR-Pita, has been cloned and characterized as a putative neutral zinc metalloprotease (23). Current evidence suggests that the predicted mature AVR-Pita metalloprotease triggers hypersensitive resistance in rice by binding to the Pi-ta C-terminal LRR region, referred to as the Pi-ta leucine-rich domain (LRD) because of the highly imperfect nature of the LRRs in Pi-ta (12). Further understanding specifically how the interaction of AVR-Pita and Pi-ta triggers resistance is a high priority.

Just as a shorter-term benefit to cloning $R$ genes is derived from tools to aid in $R$ gene deployment, a shorter-term benefit to cloning the corresponding $A V R$ gene is derived from understanding the $A V R$ gene structure in the field pathogen population. DNA fingerprinting analyses provide a genealogical framework for the blast population, which is predominantly asexual, with sets of discrete lineages, i.e., genetic families descended from a common ancestor $(6,16,33)$. Different lineages show predictable differences in pathotype structure-some lineages are associated with a single pathotype and others show a relatively complex structure with multiple pathotypes. Interestingly, lineages show uniform avirulence toward some $R$ gene or genes, raising the possibility that members of a given lineage may be limited in ability to modify $A V R$ recog- 
nition corresponding to these $R$ genes. For example, the SRL-1 lineage strains from the Colombian blast population uniformly contain avirulent alleles of AVR-Pita as determined by infection assays with $P i$-ta differential cultivars Yashiro-mochi and K1 (14), by AVR-Pita restriction fragment length polymorphism analysis, and by sequence analysis (22). Lineage exclusion breeding strategies based on pyramiding of "lineage-specific" $R$ genes, effective against all strains of a particular clonal population, may facilitate breeding durable resistance in rice $(6,16,33)$.

Katy and Drew are southern U.S. cultivars that have been used extensively as breeding parents for blast resistance (19-21). 'Katy' was produced from a cross of 'Bonnet 73'/C19722/'Starbonnet'/'Tetep'/'Lebonnet' (19). 'Drew', derived from a cross of 'Newbonnet' and 'Katy', is still grown over large acreage in the state of Arkansas (21). 'Katy' has been proposed to contain the $P i$ $t a^{2}$ gene, or both $P i$-ta and $P i-t a^{2}$ (20). There is one report that 'Katy' contains a tightly linked cluster of at least seven $R$ genes that map in the same region as $P i$-ta and $P i-t a^{2}(5,20)$. Molecular confirmation of $P i$-ta presence in 'Katy' and 'Drew' provides essential knowledge to facilitate pyramiding of this $R$ gene into advanced Arkansas breeding lines.

The Latin American rice cultivars Cica 9 and El Paso 144 lack $P i$ - $t a$ because they are uniformly susceptible to the SRL- 1 family of blast lineages, which are characterized by avirulent alleles of AVR-Pita $(6,16,22)$. 'Cica 9' is an interesting case because it is broadly resistant to all Colombian lineages except SRL-1, and SRL-1 strains are the only ones recovered from lesions on 'Cica 9' in the field. This suggests that 'Cica 9' contains many $R$ genes but not $P i$-ta $(6,16)$. El Paso 144 and related tropical indica cultivars have been widely grown in the southern cone region of South America. In the field, these cultivars are only infected by the indigenous SRL-1-related lineages that also contain AVR-Pita, and these lineages cause devastating blast disease on these cultivars in the absence of fungicide treatment (M. Levy, A. Livore, and S. Avila, personal communication). Cica 9 and El Paso 144 are two rice cultivars in which incorporation of $P i$-ta, along with their current complement of $R$ genes, would immediately impact resistance in the field.

Analysis of the natural structural range of resistance and susceptibility alleles of $R$ genes will not only benefit plant breeders in confirming the presence of an $R$ gene but will also provide insight into the molecular evolution of $R$ genes $(27,28)$. More than $30 R$ genes have been cloned and characterized, with many of these from the model plant system Arabidopsis thaliana (10). Roughly two-thirds of the cloned genes occur at complex loci containing multiple family members, or paralogs (10), and the remaining $R$ genes occur at simple loci composed of single genes. To date, only a few studies of natural allelic variation of $R$ genes have been reported $(2,4,10,11,26)$. Comparisons of orthologous genes, genes from a common ancestor in different cultivars or species, have been made for the single-copy $R$ gene loci Rpm 1 and Rps 2 in $A$. thaliana accessions and the closely related species Arabis lyrata (2). Homologous alleles of many $R$ genes can be found in the susceptible cultivars, with the exception that sequences homologous to $R p m 1$ are not present in some closely related ecotypes of $A$. thaliana $(2,8,26)$. Interestingly, resistance alleles of Rps 2 are relatively conserved, whereas susceptibility alleles are widely divergent (4).

In this study, we investigated genomic DNA polymorphisms between the indica Pi-ta allele in cultivar Yashiro-mochi and a susceptibility japonica allele. We sequenced the $\mathrm{Pi}$-ta alleles from the additional rice cultivars Katy, Drew, Cica 9, El Paso 144, IR64, and Oryzica Llanos 5. These latter two cultivars are considered to possess durable blast resistance, but little is known of their $R$ gene compositions. We verified resistance or susceptibility phenotypes with infection assays by using isogenic fungal strains that differ only in the presence or absence of AVR-Pita. Each of these new rice cultivars contains a single $P i$-ta ortholog. Relatively low lev- els of sequence diversity were found, even between the genes from indica and japonica rices. The resistance $P i$ - $t a$ allele has been confirmed in cultivars Katy and Drew. Cultivars Cica 9 and El Paso 144 identify a third susceptibility pi-ta haplotype. The functional polymorphism at amino acid 918 continues to differentiate resistant and susceptible cultivars. Understanding the natural polymorphism at the $\mathrm{Pi}$-ta locus is required for designing primers for MAS to facilitate deploying this gene into advanced breeding lines.

\section{MATERIALS AND METHODS}

Rice cultivars, fungal strains, and pathogenicity assays. Japanese differential cultivars (14) Yashiro-mochi (Pi-ta/Pi-ta) and Tsuyuake (pi-talpi-ta) were obtained from Hajime Kato (currently at Kwansei Gakuin University, Kobe, Japan). 'Yashiro-mochi' was the source of the cloned Pi-ta gene (3), and 'Tsuyuake' contains a japonica susceptibility pi-ta allele. 'Yashiro-mochi' and 'Tsuyuake' were parents of a doubled haploid rice population produced by Zaida Lentini, Cesar Martinez, and Joe Tohme at Centro Internacional de Agricultura Tropical (CIAT, Cali, Colombia), including lines YT14 (Pi-ta/Pi-ta), YT16 (pi-talpi-ta), and YT171 (Pi-ta/Pi-ta) (3). Southern U.S. rice cultivars Katy and Drew were obtained from Fleet Lee (Rice Research and Extension Center, University of Arkansas, Stuttgart, AR). El Paso 144, a tropical indica cultivar from South America, was provided by Morris Levy (Purdue University, West Lafayette, IN). Cultivars Cica 9 and Oryzica Llanos 5 (6) were provided by Cesar Martinez (CIAT). Cultivars C101A51 (17) and IR64 were provided by the International Rice Research Institute (Los Baños, Philippines).

Infection assays were performed as described (3). Briefly, plants were spray-inoculated with $5 \times 10^{4}$ spores per ml, incubated at room temperature and in low light within plastic bags for $24 \mathrm{~h}$ to maintain high humidity, and then transferred to a plant growth chamber. Humidity in the growth chamber was maintained between 70 and $85 \%$ to prevent sporulation and subsequent reinfection of susceptible plants. Disease ratings were taken 7 days postinoculation. Infection assays to confirm the presence or absence of a resistance $P i$-ta allele used the avirulent Chinese field isolate O-137 (AVR-Pita) and a spontaneous virulent mutant CP3337 (avr-pita $)^{-}$derived from $\mathrm{O}-137$ in laboratory studies as described (3). A Colombian rice pathogen (lineage SRL-1, \#13017) obtained from Dr. Morris Levy (Purdue University) was used in some assays. This strain was isolated from cultivar Cica 9 in the field and contains an avirulent $A V R$-Pita allele (22).

Construction and screening of a 'Tsuyuake' genomic library. If not specifically described, standard methods were used for library screening, DNA isolation, restriction enzyme digests, DNA blotting, and DNA blot hybridization (1). Hybridization was performed with radio-labeled ( 1 to $2 \times 10^{6} \mathrm{cpm} / \mu \mathrm{g}$ ) random hexamerlabeled polymerase chain reaction (PCR) products (7) amplified from plasmid clones. Blots were washed at $65^{\circ} \mathrm{C}$ in $0.1 \times \mathrm{SSPE}$ (1× SSPE is $0.15 \mathrm{M} \mathrm{NaCl}, 10 \mathrm{mM}$ sodium phosphate, and $1 \mathrm{mM}$ EDTA, pH 7.4) with $0.1 \%$ sodium dodecyl sulfate (SDS).

Rice genomic DNA was isolated from cultivar Tsuyuake (pi-tal $p i$-ta) by using the following procedure. Three grams of leaf tissue was ground in liquid nitrogen and mixed with $5 \mathrm{ml}$ of extraction buffer (7 M urea; 0.35 M Tris, $\mathrm{pH}$ 8.0; $0.05 \mathrm{M}$ EDTA, pH 8; 1\% Sarkosyl) and incubated at $37^{\circ} \mathrm{C}$ for $15 \mathrm{~min}$. Five milliliters of phenol/chloroform/isoamyl alcohol (25:24:1) was added and the suspension was cleared by centrifugation. One volume of isopropanol and $1 / 10$ volume of $3 \mathrm{M} \mathrm{NaOAc}(\mathrm{pH} 5.2)$ were added to the supernatant. DNA was collected by high-speed centrifugation.

A 'Tsuyuake' genomic library was constructed in the lambda ZAPII vector (Stratagene, La Jolla, CA), which allows one to avoid lambda DNA purification by in vivo excision of plasmid clones from the lambda vector. To construct this library, genomic DNA was digested with 1 unit of restriction endonuclease Tsp509I 
(New England Biolabs, Beverly, MA) at $0 \mathrm{~s}, 30 \mathrm{~s}, 1 \mathrm{~min}, 2 \mathrm{~min}, 3$ $\mathrm{min}$, and $1 \mathrm{~h}$ to determine an appropriate incubation time for partial digestion. Incubation times of $1 \mathrm{~min}, 2 \mathrm{~min}$, and $3 \mathrm{~min}$ were selected for partial digestion following visualization on an ethidium bromide-stained agarose gel. DNA fragments ranging from 7 to 10 kilobases $(\mathrm{kb})$ were purified from agarose gels by using a Qiagen extraction kit (Qiagen, Valencia, CA), following the manufacturer's protocol, and ligated to a predigested lambda ZAPII vector (Stratagene) with highly concentrated DNA ligase (Life Technologies Inc., Rockville, MD). The ligated mixture was packaged by using the Gigapack III Gold packaging extract (Stratagene), and the resulting library was amplified and screened according to the manufacturer's recommendation.

DNA probes and primers. Genomic sequence of the resistance $P i$-ta allele (GenBank accession no. AF207842) contains 2,425 base pairs (bp) of native promoter sequence, the 2,784-nucleotide $\mathrm{Pi}$-ta coding sequence interrupted by a 1,463-bp intron, and 252 bp of $3^{\prime}$ untranslated sequence (3). With pCB1641 (3) as a template for PCR, oligonucleotides F8-5 (5'-TCCTCAGAGGCGATCTCC-3') and F12-5 (5'-CGAACGGCGCATCCAACC-3') were used to amplify a DNA fragment comprising nucleotides 2,571 to 3,210 of the GenBank Pi-ta sequence (nucleotides 1,401 to 2,040 from the insert in pCB1641). The amplified fragment encodes amino acids 50 to 262 near the amino terminus of the Pi-ta protein. The resulting PCR fragment was purified by using a Qiagen kit for labeling. The probe for the $3^{\prime}$ region was a 1.1-kb EcoRI/SalI DNA fragment purified from plasmid pCB1645 encoding the C-terminal LRD comprising amino acids 586 to $928(3,12)$. The hybridization probe for screening the 'Tsuyuake' genomic library to isolate pi-ta was obtained from pCB1641 by digestion with restriction endonuclease BamHI. Resulting 2.5- and 4.5-kb fragments containing the $\mathrm{Pi}$-ta genomic region were purified for use as probes. The gene fragment encoding LRD was identified in clones from the 'Tsuyuake' genomic library by using the primers GB47 (5'-AATGCAGAATTCACAACACCACTAGCAGGTTTG-3') and GB46 (5'CATTAAAGTCGACCTCAAACAATCAAGTCAGGT-3'), containing EcoRI and SalI sites, respectively (underlined), used for subcloning this fragment into pCB1645 (3).

DNA sequence analysis. Genomic DNAs were purified from additional rice cultivars by essentially the same procedure described for 'Tsuyuake' above. The coding regions of additional $\mathrm{Pi}$-ta genes were determined by sequencing PCR products, which had been amplified by using genomic DNA as a template and purified by using a Qiagen labeling kit according to manufacturer's directions. The primers used for sequencing are listed in Table 1. DNA sequences were determined from double-stranded plasmid DNA by using an ABI 377 sequencer (Applied Biosystems, Foster City,

TABLE 1. DNA primers used for sequencing analysis in this study

\begin{tabular}{ll}
\hline Primer & \multicolumn{1}{c}{ DNA sequence } \\
\hline B7-1 & 5'-GTCAATTGTTTTTCAGTGGAG-3' \\
B7-2 & 5'-CTGTCGTCTGAGAATGACC-3' \\
F8-5 & 5'-TCCTCAGAGGCGATCTCCG-3' \\
F8-1 & 5'-GAACAGGGTGACCTCGGCT-3' \\
F12-1 & 5'-GTGGCTTCCATTGTTGGATC-3' \\
F12-2 & 5'-TGTGCTCTGAGTTATATCCAC-3' \\
F12-3 & 5'-GCTGGCTACAGATCTGTAGG-3' \\
F12-4 & 5'-CCTACAGATCTGTAGCCAGC-3' \\
F12-5 & 5'-TCGAACGGCGCATCCAACC-3' \\
$1641-3$ & $5^{\prime}$-GGGCCTCCCTTGTTCGG-3' \\
$1641-5$ & $5^{\prime}$-CCAGTCCATTTGGGGATGC-3' \\
$173-7$ & 5'-TTGTATATCAACCATAAGAGTGC-3' \\
C10-1 & 5'-CCAAGGACTACAACACTTGC-3' \\
$173-5$ & 5'-AGCATCCCCAAATGGACTGG-3' \\
$1641-6$ & 5'-GTTCTTTGATCCAAGTGTTAGG-3' \\
YL80 & 5'-CAGGATGACCTTGACACTCT-3' \\
YL81 & 5'-GGGTTGGAACACTTGGTCGC-3' \\
YL82 & 5'-AGAGACTTGGATGAAGATTT-3' \\
YL84 & 5'-GCGACCAAGTGTTCCAACCC-3' \\
\hline
\end{tabular}

CA). The DNA sequence was analyzed by using the Vector NTI suite (InforMax, North Bethesda, MD). Alignments with other DNA sequences were performed by using Vector NTI align X.

\section{RESULTS}

$P i$-ta is a single-copy gene in diverse rice cultivars. The $P i$-ta gene was previously mapped near the centromere of chromosome 12 , isolated by positional cloning and verified by genetic complementation via stable rice transformation (3). DNA gel blot analysis by using the DNA probe encoding the amino terminal portion of the Pi-ta protein identified single bands against a general smear
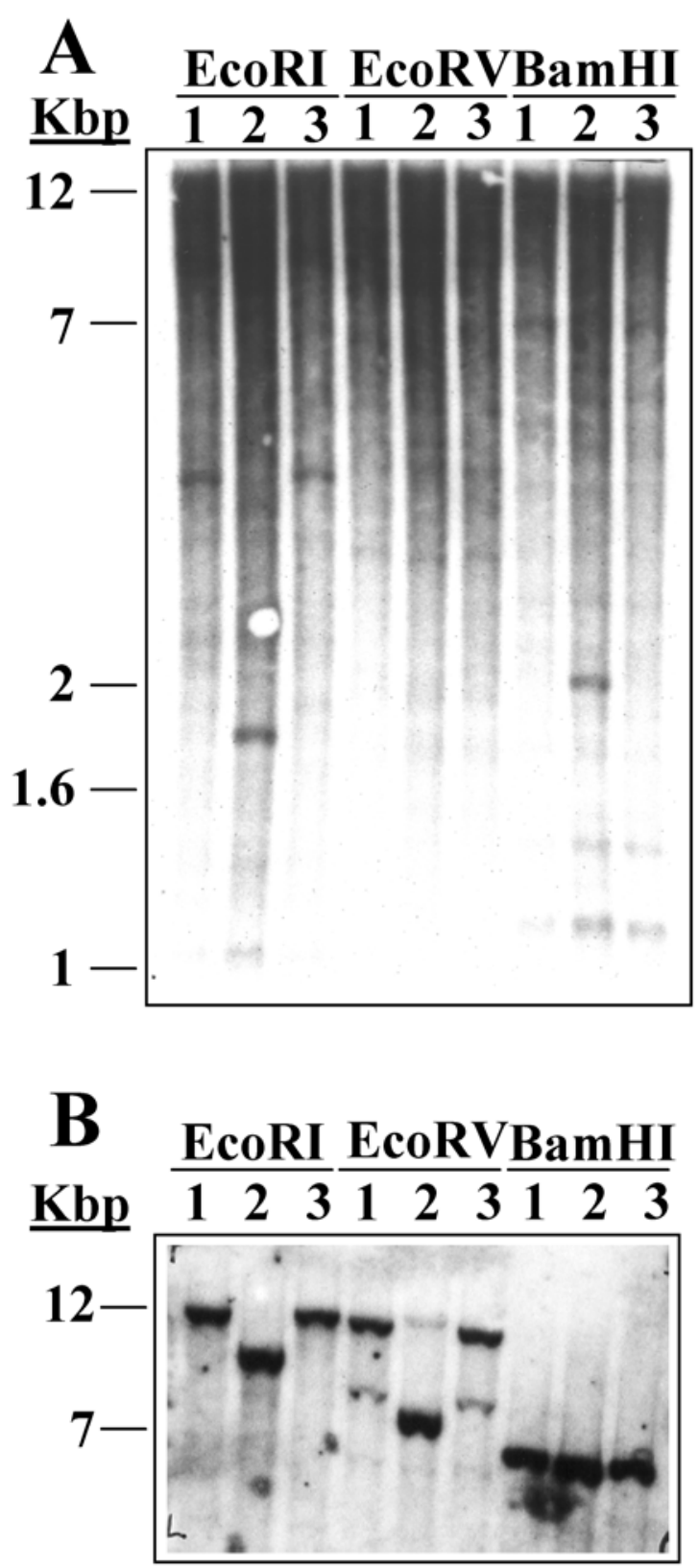

Fig. 1. DNA gel blot analysis detects a single gene in resistant and susceptible rice. Genomic DNAs (4 $\mu \mathrm{g}$ per lane) of three doubled haploid progeny used in mapping and cloning Pi-ta (3) were digested with indicated restriction enzymes. YT14 (lane 1, Pi-ta/Pi-ta) and YT171 (lane 3, Pi-ta/Pi-ta) contain linked DNA markers indicating that this region originated from the indica donor. YT16 (lane 2, pi-ta/pi-ta) contains the linked markers from the japonica allele. Samples were separated by electrophoresis in $1 \%$ agarose gels, blotted onto Hybond $\mathrm{N}^{+}$membrane, and hybridized with a radiolabeled DNA fragment from the $\mathbf{A}, 5^{\prime}$ region and $\mathbf{B}, 3^{\prime}$ region of the $\mathrm{Pi}-\mathrm{ta}$ coding sequence. $\mathrm{Kpb}=$ kilobase pairs. 
of background hybridization (Fig. 1A). This suggests the presence of a large number of related DNA sequences in the genome in both resistant and susceptible rice lines. The DNA blot was then stripped to remove the hybridization signals and probed with the 3' DNA fragment encoding the Pi-ta LRD region (Fig. 1B). Only one hybridization band appeared after digestion with EcoRI and BamHI, which is consistent with the lack of these restriction sites in the DNA fragment used as the probe. EcoRV, which cleaves the probe fragment once, produced two homologous bands, as expected for a single-copy gene. DNA gel blot analysis with the $3^{\prime}$ probe identified a single $P i$-ta band in diverse rice cultivars (Fig. 2). Thus, both Pi-ta and pi-ta appear to be single-copy genes in all of these rice cultivars. The only polymorphisms detected by these analyses occurred between indica and japonica alleles (Figs. 1 and 2).

Cloning and analysis of a susceptibility pi-ta homolog from japonica rice. Eight lambda clones from a library of 'Tsuyuake' genomic DNA hybridized to the full-length insert of the Pi-ta gene. A DNA fragment approximately $1 \mathrm{~kb}$ in length was amplified from six of the eight lambda lysates by using DNA primers GB46 and GB47, suggesting that they are DNA fragments corresponding to the $3^{\prime}$ end of the coding region of the Pi-ta gene. No amplification was observed from the lambda lysates $\lambda \mathrm{T} 2-1$ and $\lambda T 2-9$, suggesting that they contain a different region of the Pi-ta gene (Fig. 3A). To confirm this, in vivo excision was performed to obtain plasmid clones corresponding to each lambda lysate. All plasmid DNA was isolated and digested with the restriction enzyme EcoRI. Digested DNA was separated by gel electrophoresis, stained with ethidium bromide, and visualized with ultraviolet light (Fig. 3B, left). The gel was then blotted and probed with a radioactively labeled probe from the $5^{\prime}$ translation start region. Blot analysis and sequencing confirmed that the inserts in plasmids T2-2 and T2-9 overlap by $600 \mathrm{bp}$ in the middle of the pi-ta gene and contain the largest stretch of flanking sequences at the pi-ta locus (Fig. 3C).

The T2-2 and T2-9 plasmid inserts were sequenced to produce a 5,113-bp DNA sequence. Similar to the dominant $P i$-ta allele, $p i$ ta encodes an open reading frame (ORF) interrupted by a similarly sized intron and predicted to encode a protein with 928 amino acids (Fig. 4). The ATG that initiates this ORF occurs

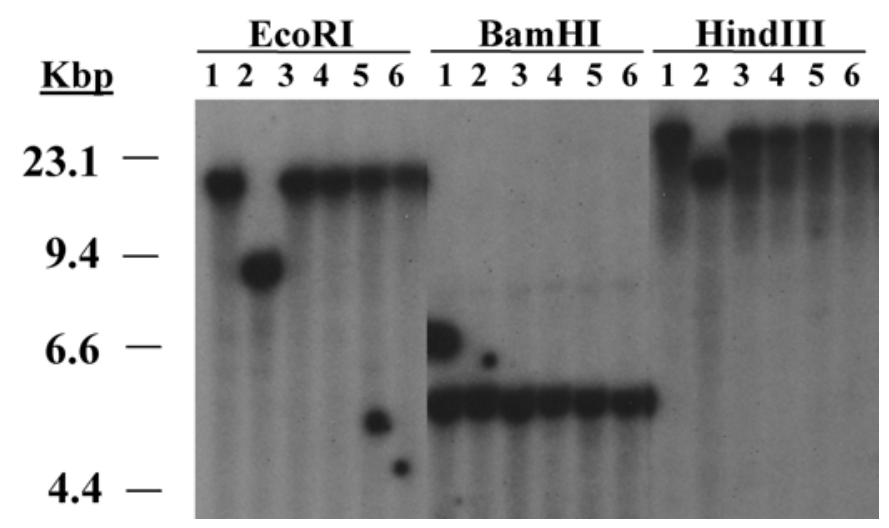

Fig. 2. DNA gel blot analysis of the Pi-ta locus from diverse rice cultivars. Genomic DNAs ( $4 \mu \mathrm{g}$ per lane) were digested with indicated restriction enzymes. Samples were separated by electrophoresis in $0.7 \%$ agarose gels, blotted onto Hybond $\mathrm{N}^{+}$membrane, and hybridized with the radio-labeled probe from the $3^{\prime}$ region of the Pi-ta coding sequence (3). Rice cultivars from the left are Yashiro-mochi, Tsuyuake, C101A51, Cica 9, IR64, and Oryzica Llanos 5, lanes 1 to 6 , respectively. The japonica cultivar Yashiro-mochi obtained its Pi-ta gene from an indica source and japonica cultivar Tsuyuake contains the japonica pi-ta allele. All other cultivars are indica. Cultivars Yashiro-mochi, Tsuyuake, C101A51, and Cica 9 each represent one of the four Pi-ta haplotypes identified by sequence analysis. Extra spots in lanes 5 and 6 of the EcoRI digest and lanes 1 and 2 of the BamHI digest result from background noise in this particular autoradiograph. $\mathrm{Kpb}=$ kilobase pairs. within a favorable context for translation initiation, for example, with guanine at +4 (15). The DNA sequence of the $p i$-ta gene was aligned with the Pi-ta gene by Vector NTI align X. Overall, the DNA sequence of the japonica pi-ta gene is $99.6 \%$ identical to $P i$-ta. The intron in the pi-ta gene is $99.3 \%$ identical to the intron in $P i$-ta (Fig. 4; Table 2). A single nucleotide difference was identified in both the $5^{\prime}$ leader region and the $3^{\prime}$ noncoding regions (Table 2). The pi-ta and Pi-ta genes differ by five nucleotide substitutions (all transversions) in the first exon and two nucleotide substitutions (one transition and one transversion) in the second exon. Thus, the most conserved region of the gene is the second exon, which encodes the LRD.

The Pi-ta and pi-ta coding sequences differ by five nonsynonymous changes resulting in amino acid differences and two synonymous nucleotide changes not resulting in amino acid substitutions (Fig. 5; Table 2). Pi-ta and pi-ta encode putative cytoplasmic proteins with four amino acid differences located in the $\mathrm{NH}^{2}$ terminus, with an identical NBS region, and with a single amino acid substitution in the LRD at the carboxyl terminus.
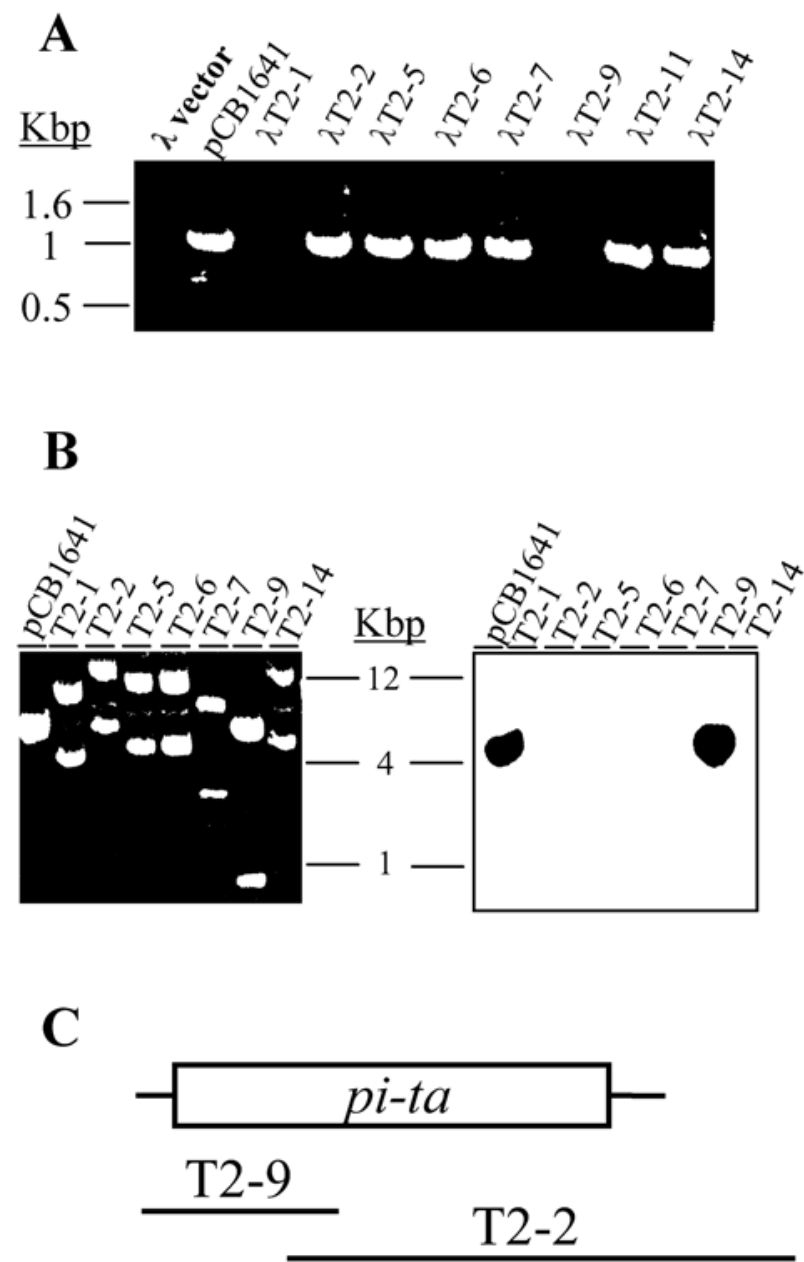

Fig. 3. Identification of overlapping genomic clones spanning the pi-ta gene. A, Identification of lambda lysates containing the $3^{\prime}$ region of the pi-ta gene. Purified lambda lysates were amplified by polymerase chain reaction with $3^{\prime}$ $P i$-ta gene-specific primers. pCB1641 contains the $P i$-ta gene (3) and was used as a positive control; the lambda vector was used as a negative control. Lambda lysates T2-2, T2-5, T2-6, T2-7, T2-11, and T2-14 contained sequences corresponding to the $3^{\prime}$ region of Pi-ta. B, Identification of a plasmid clone that contains the $5^{\prime}$ region of the $P i$-ta gene. Plasmid DNA was digested with EcoRI, separated by agarose gel electrophoresis, and visualized by ethidium bromide staining (right). The gel was blotted onto Hybond $\mathrm{N}^{+}$ membrane and hybridized with the radio-labeled DNA fragment from the 5' region of the Pi-ta coding sequence (the same probe as in Fig. 1B). Plasmid pCB1641 was used as a positive control. C, Overlapping plasmid clones spanning the pi-ta region. $\mathrm{Kpb}=$ kilobase pairs. 
Sequence analyses and pathogenicity testing of additional $P i$-ta alleles. Sequencing of $P i$-ta from resistant rice cultivars Katy and Drew identified alleles identical to Pi-ta from 'Yashiromochi'. The susceptibility pi-ta coding sequence from indica cultivar C101A51 differs by two nucleotides from the 'Yashiro-mochi' coding sequence, a synonymous change at nucleotide 384 and a nonsynonymous change at nucleotide 2,752 (Table 3). Sequencing of pi-ta from susceptible indica rice cultivars Cica 9 and El Paso 144 identified an additional susceptibility allele, which differs from the 'C101A51' allele by including the nonsynonymous substitution at nucleotide 17. A blast search of the publicly available rice genome sequence of the Chinese indica cultivar 93-11 (31), deposited with the Beijing Genomics Institute (Rice GD), identified a sequence identical to the susceptibility pi-ta allele in 'Cica 9' and 'El Paso 144'. Sequencing the alleles in the durably resistant cultivars IR64 and Oryzica Llanos 5 gave both alternative nucleotides at positions 17,384 , and 2,752, indicating the presence of both resistance and susceptibility alleles, probably because of seed mixtures. No new changes were identified from sequencing new $P i$-ta alleles. The nucleotide substitutions corresponding to susceptibility indica alleles are a subset of those previously identified in the japonica allele.

Pathogenicity tests were performed using rice pathogens with an AVR-Pita or an avr-pita allele in order to confirm Pi-ta speci-
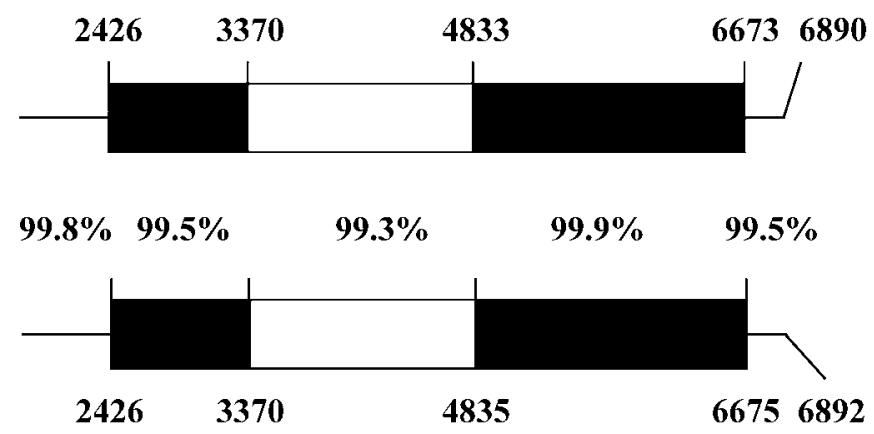

Fig. 4. Physical maps of the Pi-ta region in 'Yashiro-mochi' and 'Tsuyuake'. Genomic regions from resistant cultivar Yashiro-mochi containing the Pi-ta region (top) and susceptible cultivar Tsuyuake containing the pi-ta region (bottom) are shown. The open reading frames of Pi-ta and pi-ta are shown in solid black. Introns are indicated by the open box. Percentages of identical nucleotides for selected regions are presented in the middle. The numbers above $P i$-ta and below pi-ta are the nucleotide numbers corresponding to the $P i$-ta genomic sequence in GenBank (accession no. AF207842). Comparisons for calculation of identity percentages were determined by using the Vector NTI suite. The genomic nucleotide sequence of the japonica pi-ta gene has been submitted to GenBank as accession no. AY196754. ficity (Table 4). Strain O-137 contains an avirulence allele of AVR-Pita, while strain CP3337 is an isogenic virulent mutant derived from O-137. In the absence of interfering $R$ genes, the pattern of resistance to avirulent strain O-137 and susceptibility to its isogenic virulent mutant CP3337 can define the presence of Pi-ta. For example, rice cultivars Yashiro-mochi, Katy, Drew, and IR64 are shown to contain $\mathrm{Pi}$-ta because they are resistant to O-137 but susceptible to CP3337. In contrast, rice cultivars C101A51, El Paso 144, and YT16 are susceptible to both strains. Pathogenicity tests with O-137 and CP3337 give no information on the presence of Pi-ta in 'Cica 9' or 'Oryzica Llanos 5'. The resistance reaction with both $\mathrm{O}-137$ and $\mathrm{CP} 3337$ indicate that some other $R$ gene is masking the gained virulence phenotype associated with mutant CP3337. The presence of the susceptibility pi-ta allele in 'Cica 9' was confirmed in infection assays with the avirulent Colombian SRL-1 strain (data not shown). Oryzica Llanos 5, however, is a highly resistant cultivar and is not susceptible to any of these strains. Seed from the sequenced Chinese indica cultivar 93-11 was not available for pathogenicity assays.

\section{DISCUSSION}

Each polymorphism differentiating susceptibility and resistance alleles provides an opportunity for developing reliable molecular markers for incorporating Pi-ta into advanced breeding lines. Particularly valuable are functional polymorphisms that are directly responsible for the resistance or susceptibility phenotypes. This study further strengthens our previous report (3) of a functional polymorphism at $P i$-ta nucleotide 2,752, which results in an alanine at amino acid 918 in the resistance Pi-ta protein or a serine at this location in the susceptibility pi-ta protein. DNA markers that identify this functional polymorphism eliminate the potential for recombination associated with conventional linked molecular markers.

The Pi-ta and $P i$ - $t a^{2}$ genes commonly used in rice breeding programs worldwide have originated from several traditional indica cultivars, including Tetep from Vietnam and Tadukan from the Philippines. The Pi-ta gene from 'Yashiro-mochi' was derived from an upland rice cultivar, Okaine (24). Resistance alleles from all three sources are identical. The Pi-ta alleles in 'Katy' and 'Drew' were derived from 'Tetep' (19-21), and they encode proteins identical to all other resistance Pi-ta proteins (3). Genome sequence from the japonica susceptibility pi-ta allele identified seven nucleotide differences relative to the indica Pi-ta coding sequence. Susceptibility alleles from indica cultivars contain a subset of these differences. Cultivars Cica 9 and El Paso 144 contain a new susceptibility allele, which has a nonsynonymous change at nucleotide 17 in addition to the substitutions at nucleotides 384

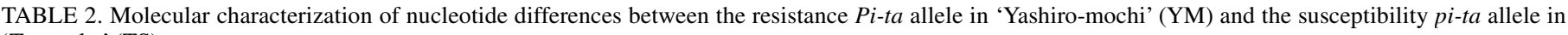
'Tsuyuake' (TS)

\begin{tabular}{|c|c|c|c|c|}
\hline Cultivars & Position $^{\mathrm{a}}$ & Nucleotide change & Amino acid change and position & Type of mutation \\
\hline YM to TS & 2,040 & $\mathrm{G}$ to $\mathrm{A}$ & $5^{\prime}$ noncoding sequence & Transition \\
\hline YM to TS & $2,442(17)$ & $\mathrm{T}$ to $\mathrm{G}$ & Ile 6 Ser & Transversion \\
\hline YM to TS & $2,809(384)$ & $\mathrm{G}$ to $\mathrm{C}$ & No change & Transversion \\
\hline YM to TS & $2,839(444)$ & $\mathrm{C}$ to $\mathrm{G}$ & Arg 148 Ser & Transversion \\
\hline YM to TS & $2,899(474)$ & $\mathrm{C}$ to $\mathrm{G}$ & His $158 \mathrm{Gln}$ & Transversion \\
\hline YM to TS & $2,952(527)$ & A to $\mathrm{T}$ & Asp $176 \mathrm{Val}$ & Transversion \\
\hline YM to TS & 3,536 & $\mathrm{~T}$ to $\mathrm{CC}$ & Intron & Insertion/deletion \\
\hline YM to TS & 4,234 & $\mathrm{G}$ to $\mathrm{A}$ & Intron & Transition \\
\hline YM to TS & 4,270 & $\mathrm{G}$ to $\mathrm{A}$ & Intron & Transition \\
\hline YM to TS & 4,391 & $\mathrm{C}$ to $\mathrm{T}$ & Intron & Transition \\
\hline YM to TS & 4,394 & $\mathrm{~T}$ to $\mathrm{A}$ & Intron & Transversion \\
\hline YM to TS & $4,426-4,428$ & GCC to CTAT & Intron & Insertion/deletion \\
\hline YM to TS & $6,276(2,388)$ & $\mathrm{G}$ to $\mathrm{A}$ & No change & Transition \\
\hline YM to TS & $6,640(2,752)$ & $\mathrm{G}$ to $\mathrm{T}$ & Ala 918 Ser & Transversion \\
\hline YM to TS & 6,808 & $\mathrm{~T}$ to $\mathrm{A}$ & $3^{\prime}$ noncoding sequence & Transversion \\
\hline
\end{tabular}

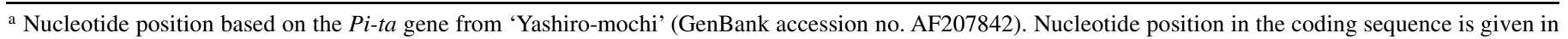
parentheses. 
and 2,752 in the previously reported 'C101A51' susceptibility allele. We have not yet clarified the anomalous sequencing results suggesting that 'IR64' and 'Oryzica Llanos 5' contain both resistance and susceptibility alleles. Since the genomic DNAs used in the PCR sequencing analysis were produced from multiple plants of each cultivar, the most likely explanation for this is impure seed. Still, this analysis tells us that the 'IR64' and 'Oryzica Llanos 5' Pi-ta genes do not contain novel amino acid substitutions relative to other sequenced genes.

As originally recognized in the 1970s by Kiyosawa (13), pathogenicity assays with avirulent field isolates and virulent mutants obtained from these strains are more informative than are assays with the avirulent strain alone. Resistance to the avirulent strain and susceptibility to its corresponding virulent mutant identifies the corresponding $R$ gene in a particular rice cultivar. Our pathogenicity testing was performed using a Chinese field isolate O137 with AVR-Pita and an isogenic laboratory mutant CP3337 with a virulence pi-ta allele. Infection assays confirm the occurrence of Pi-ta in cultivars Katy, Drew, and IR64, and they confirm the susceptibility allele in cultivars El Paso 144 and C101A51. Infection with these strains failed to determine whether cultivars Cica 9 and Oryzica Llanos 5 contain resistance or susceptibility alleles of Pi-ta, because the avr-pita mutant CP3337 remains avirulent on these cultivars. Strains O-137 and CP3337 apparently contain at least one additional $A V R$ gene corresponding to another $R$ gene in each of these cultivars. Among the 13 AVR genes identified by the Japanese differential rice cultivars (14), O-137 contains only $A V R-P i-z^{t}$ in addition to AVR-Pita (B. Valent, unpub-

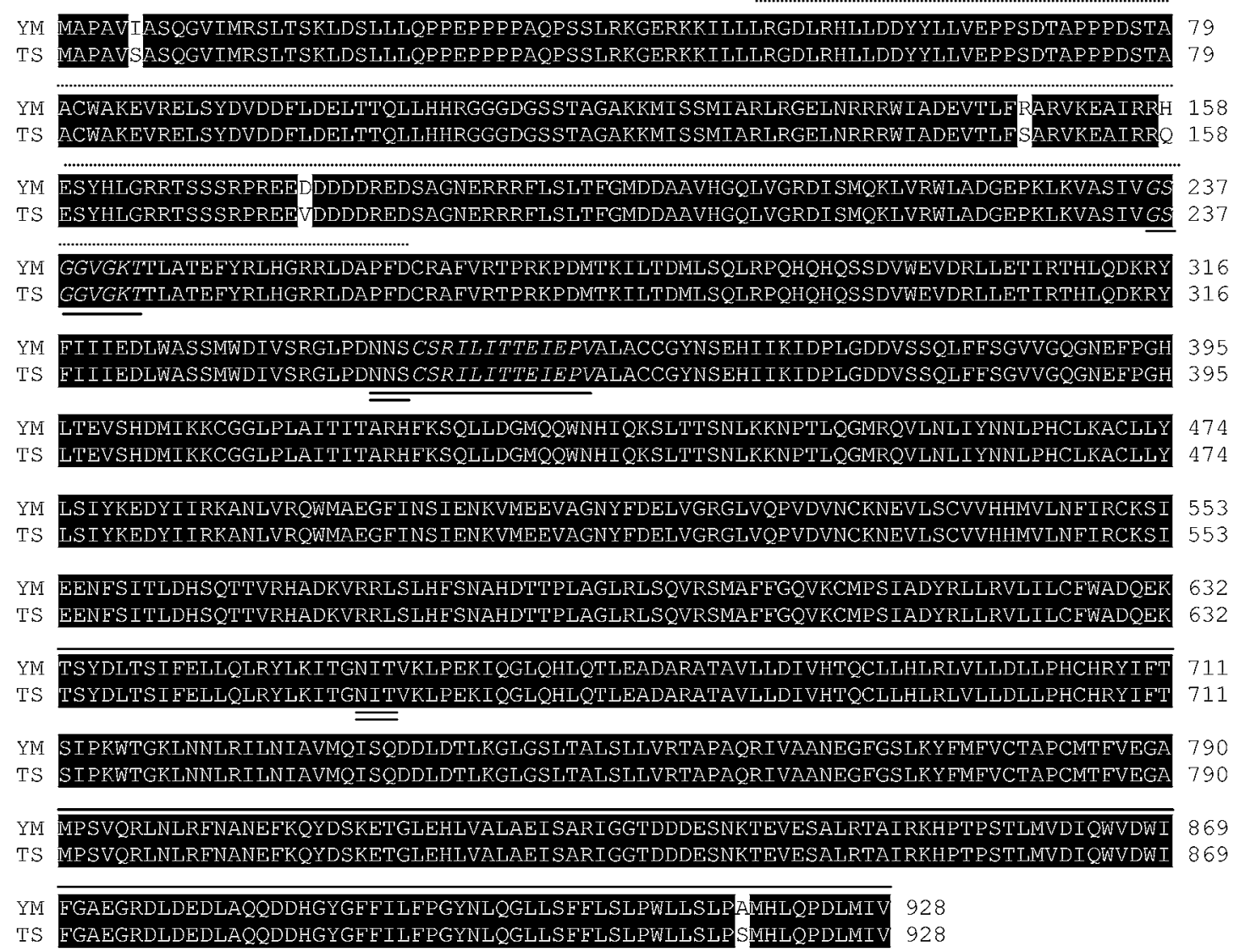

Fig. 5. Alignment of the amino acids in the predicted indica Pi-ta and japonica pi-ta proteins. Identical amino acids are shown in black boxes. Predicted susceptibility indica alleles contain only the A918S substitution or both the I6S and A918S substitutions. Alignment was produced by using align X of the vector NTI program. The putative nucleotide binding site motifs are shown in italics and underlined. They are amino acids 236 to 244 , which fit the generalized consensus GxGxxG(R/K) for a phosphate binding (P) loop; amino acids 314 to 323, the probable kinase 2 motif; and amino acids 342 to 354 , the probable kinase 3a motif. Potential N-glycosylation sites are double-underlined. The N-terminal region encoded by the 5 ' hybridization probe (Figs. 1 and 3 ) is shown with a dotted line over the amino acids. The $\mathrm{C}$-terminal leucine-rich domain region is shown with a solid line over the amino acids. YM = cultivar Yashiro-mochi and TS = cultivar Tsuyuake.

TABLE 3. Naturally occurring Pi-ta haplotypes

\begin{tabular}{lcccccc}
\hline & \multicolumn{5}{c}{ Nucleotide position $^{\mathrm{a}}$} \\
\cline { 2 - 7 } Allele source & 17 & 384 & 444 & 474 & 527 & 2,388 \\
\hline Yashiro-mochi $^{\mathrm{b}}$ & $\mathrm{T}$ & $\mathrm{G}$ & $\mathrm{G}$ & $\mathrm{C}$ & $\mathrm{A}$ & $\mathrm{G}$ \\
C101A51 & $\mathrm{T}$ & $\mathrm{C}$ & $\mathrm{G}$ & $\mathrm{C}$ & $\mathrm{A}$ & $\mathrm{G}$ \\
El Paso 144c $^{\text {Tsuyuake }}$ & $\mathrm{G}$ & $\mathrm{C}$ & $\mathrm{G}$ & $\mathrm{C}$ & $\mathrm{A}$ & $\mathrm{T}$ \\
\hline
\end{tabular}

${ }^{a}$ Nucleotide position in the cDNA coding sequence. Polymorphisms at nucleotides 17, 444, 474, 527, and 2,752 result in amino acid substitutions.

b Pi-ta-containing rice cultivars K1, Reiho, Tetep, Tadukan, Katy, and Drew contain this allele.

${ }^{c}$ pi-ta-containing indica rice cultivar Cica 9 also contains this allele. The sequenced Chinese indica cultivar 93-11 contains this allele and is, therefore, predicted to contain the susceptibility pi-ta allele.

${ }^{\mathrm{d}}$ pi-ta-containing japonica rice cultivars Nipponbare and Sariceltik contain this allele (3). 
lished data). Thus, we have found these strains to be generally useful for detecting $P i$-ta in the presence of many other $R$ genes. However, even relatively well-characterized $M$. grisea strains can be expected to contain additional $A V R$ genes that mask identification of a particular $R$ gene in the cultivar of interest. Molecular cloning and characterization of more $A V R$ genes corresponding to agronomically valuable $R$ genes would provide valuable tools for defining $R$ gene composition in donor rice cultivars.

We previously reported that $P i-t a^{2}$-containing rice cultivars, such as Reiho, uniformly contain $P i$-ta as well as a second $R$ gene identified by a second $A V R$-vir mutant pair of strains lacking $A V R$ Pita homology altogether (3). Interestingly, 'Katy' and 'Drew', which are both reported to contain $P i-t a^{2}(20)$, each contain this same additional $R$ gene identified in $P i-t a^{2}$ cultivars studied previously (3; B. Valent, unpublished data). Thus, our data continue to suggest that the $P i-t a^{2}$ specificity spectrum results from action of at least two $R$ genes.

$R$ gene polymorphism is likely to be an important component of the large variation in plant species and their abilities to resist pathogens $(2,4,10,26)$. Comprehensive studies of $R$ genes that confer resistance in A. thaliana to the bacterial pathogen Pseudomonas syringae suggest that $R$ genes have a wide range of evolutionary ages. Levels of nucleotide polymorphism at synonymous sites, or in noncoding regions, reflect the ages of alleles. In general, synonymous nucleotide substitutions (Ks) that do not alter the protein product accumulate to a greater extent in protein coding sequences than do nonsynonymous substitutions (Ka) that alter the protein product. Therefore, ratios of $\mathrm{Ka}>\mathrm{Ks}$ may provide evidence for positive selection for amino acid divergence. Detailed analyses have been reported for Rps 2 and Rpml and their orthologs from different Arabidopsis ecotypes or species. Some closely related Arabidopsis ecotypes did not contain orthologs of the Rpml gene (8). Sequence analysis of the junction region flanking the Rpm 1 deletion revealed approximately $10 \%$ divergence between resistant and susceptible lineages, suggesting relatively ancient divergence of alleles that may have been maintained through a balancing selection mechanism (26). This would suggest that Rpml is selectively deleterious to the plant such that the deletion of the $R$ gene is advantageous in the absence of pathogen with the corresponding AVR gene (2). Recently, Tian et al. (28) reported the first experimental evidence suggesting that a large cost of $R P M 1$ contributes to the maintenance of an ancient $R$ gene polymorphism. A relatively high level of nucleotide polymorphisms (1.2\%) was found at the Rps2 locus, with 19 synonymous changes, 16 amino acid replacements, and 1 nonsense mutation. Resistance alleles of Rps 2 are more conserved in structure than are susceptibility alleles. It has been suggested that the Rps 2 locus

TABLE 4. Differential susceptibility to an avirulent AVR-Pita strain and its virulent avr-pita mutant identifies $P i$-ta

\begin{tabular}{llccc}
\hline Cultivar & \multicolumn{1}{c}{ Origin } & $\begin{array}{c}\text { Reaction to } \\
\text { O-137 }\end{array}$ & $\begin{array}{c}\text { Reaction to } \\
\text { CP3337 }\end{array}$ & $\begin{array}{c}P i-t a \\
\text { allele }\end{array}$ \\
\hline Yashiro-mochi & Japan (24) & $\mathrm{R}^{\mathrm{c}}$ & $\mathrm{S}^{\mathrm{c}}$ & $P i-t a$ \\
Katy & Southern U.S. (19) & $\mathrm{R}$ & $\mathrm{S}$ & $P i-t a$ \\
Drew & Southern U.S. (21) & $\mathrm{R}$ & $\mathrm{S}$ & $P i$-ta \\
El Paso 144 & South America, & & & \\
& $\quad$ Southern cone region & $\mathrm{S}$ & $\mathrm{S}$ & $p i-t a$ \\
Cica 9 & CIAT (6,31) & $\mathrm{R}$ & $\mathrm{R}$ & $?^{\mathrm{d}}$ \\
C101A51 & IRRI (17) & $\mathrm{S}$ & $\mathrm{S}$ & $p i-t a$ \\
YT16 & This study (3) & $\mathrm{S}$ & $\mathrm{S}$ & $p i-t a$ \\
IR64 & IRRI & $\mathrm{R}$ & $\mathrm{S}$ & $P i-t a$ \\
Oryzica Llanos 5 & CIAT (6,31) & $\mathrm{R}$ & $\mathrm{R}$ & $?$ \\
\hline
\end{tabular}

${ }^{\mathrm{a}}$ This Chinese field isolate was the source of the cloned AVR-Pita gene (23).

b A spontaneous mutant of strain O-137 that lost function of AVR-Pita, obtained in laboratory studies as described (3).

${ }^{c}$ Infection assays and scoring of resistant and susceptible interactions have been described (3).

${ }^{\mathrm{d}}$ Cica 9 was determined to have pi-ta by its susceptibility to a different strain containing AVR-Pita (details in text). also contains old alleles that may be the product of balancing selection (4). In contrast, the two resistance and one susceptibility alleles examined for the Rps 4 gene from A. thaliana contain relatively low levels of polymorphism (2), similar to Pi-ta. Allelic diversity at the Rps 4 locus has not yet been extensively studied.

The study of DNA polymorphisms at the Pi-ta locus provides insight into the molecular evolution of the Pi-ta gene in response to populations of the agronomically important rice blast fungus. All rice cultivars tested have a $P i$-ta ortholog, and only a few nucleotide polymorphisms occur between the resistance $P i$ - $t a$ allele and susceptibility pi-ta alleles. The highest levels of sequence diversity (approximately $0.7 \%$ ) occur in the intron (Fig. 4). The rate of nucleotide polymorphism observed in the intron is consistent with the average diversity rates of $0.64 \%$ between indica and japonica rices (31). The single nucleotide polymorphism (SNP) rates between the indica and japonica $P i$-ta intron sequence is $0.55 \%$ (eight nucleotides in the 1,463-bp intron; Table 2) compared with an average SNP rate of $0.50 \%$. The insertion-deletion polymorphism (InDel) rate in the intron is $0.14 \%$ (two nucleotides in the 1,463-bp intron; Table 2) compared with an average InDel rate of $0.14 \%$. In contrast, the two $P i$-ta exons exhibit 0.5 and $0.1 \%$ total polymorphisms, respectively.

Different domains in a protein can be subject to differing degrees of divergent selection. Ka-to-Ks ratios can be considered separately for differences between the indica resistance allele and the japonica susceptibility allele in three distinct regions of the $P i$-ta coding sequence. The N-terminal region has four nonsynonymous and one synonymous nucleotide substitution, the internal NBS region has no nucleotide substitutions, and the LRD region has one nonsynonymous and one synonymous substitution. Thus, it might be concluded that only the $\mathrm{N}$-terminus encoding region of $P i$ - $t a$ shows evidence of diversifying selection. Indeed, the background smear of hybridization seen in Southern analysis with the $\mathrm{N}$-terminal Pi-ta probe suggests that there are multiple genes with related $\mathrm{N}$-terminal sequences. Among other $R$ genes characterized, only the flax rust $L$ gene shows evidence for diversifying selection in the N-terminal domain (10). Evidence for diversifying selection is generally evident in the C-terminal LRRs, which are believed to be involved in protein-protein interactions. Indeed, we have evidence suggesting that the Pi-ta LRD region is involved in proteinprotein interactions with its corresponding AVR-Pita avirulence protein (12). However, this region of the Pi-ta protein contains only the functional polymorphism at amino acid 918. Thus, Pi-ta shows opposite patterns for diversifying selection relative to most other $R$ genes (10). High levels of conservation of gene structure might indicate DNA polymorphisms that were recently evolved, although the normal levels of diversity in the intron might argue against this. Alternatively, balancing selection may preserve relatively ancient alleles if each has faced strong selection for function.

Numerous observations suggest that $R$ genes may not be advantageous to the organism in the absence of the pathogen. For example, rice cultivars in California have lost blast resistance genes (for example $P i-k^{h}$ ) after years of breeding efforts in the absence of $M$. grisea (J. Oster, personal communication). The frequency of the corresponding $A V R$ gene in the pathogen population determines the selective advantage of maintaining an $R$ gene allele. The fact that pi-ta is also predicted to encode an NBS-LRR protein suggests that pi-ta may confer resistance to $M$. grisea pathotypes or other pathogens. Alternatively, the Pi-ta and pi-ta alleles may contain low levels of polymorphisms because they are involved in other physiological responses in rice, although alternative functions have not yet been described for any $R$ gene (10). Sequences related to $P i$-ta discovered in rye, barley, and other grass species suggest that their functions are similar to the Pi-ta gene within and across species (3). However, biological functions for $p i$-ta and for $P i$-ta orthologs in other crop species remain to be determined.

Blast resistance provided by 'Katy' or 'Drew' has been effective in the southern United States since their release, in spite of viru- 
lent $M$. grisea isolates that have been recovered from the field $(19,21$; J. C. Correll, personal communication). This study demonstrates that the Pi-ta gene is one component of the resistance in both 'Katy' and 'Drew'. It is of interest to characterize the AVRPita alleles in the virulent field isolates that have gained the ability to infect 'Katy' and 'Drew'.

Previous RNA blot analysis (3) indicates that there were no differences in transcript size or abundance produced by the resistance $P i$ - $t a$ gene and the japonica pi-ta gene. A single amino acid difference between the Pi-ta and pi-ta proteins apparently differentiates their functions. Research is underway to determine how this amino acid difference accounts for the difference between resistance and susceptibility to AVR-Pita-containing strains of the rice blast fungus. The pi-ta protein was impaired in its ability to interact with putative mature AVR-Pita protease in the yeast two-hybrid system and in vitro binding studies (12). Thus, we hypothesize that the inability of pi-ta to interact with AVR-Pita may explain why plants expressing pi-ta are not resistant to $M$. grisea strains expressing AVR-Pita. Understanding the exact molecular nature of the recognition event between Pi-ta and AVR-Pita may lead to strategies to broaden $\mathrm{Pi}$-ta recognition specificity and to more reliably trigger resistance in the presence of the rice blast pathogen.

\section{ACKNOWLEDGMENTS}

We thank M. H. Jia for proofreading; A. L. Blas, L. Imboden, and Z. Wang for technical assistance. We also thank Y. Yang, J. N. Rutger, G. Thompson, R. S. Zeigler, and anonymous reviewers for a critical reading of the manuscript. We acknowledge the DuPont DNA sequence facility and the genomic core facility of USDA-ARS, Dale Bumpers National Rice Research Center (DB NRRC), for determining all DNA sequences.

\section{LITERATURE CITED}

1. Ausubel, F. M., Brent, K., Kingston, R. E., Moore, D. D., Smith, J. A., Seidman, J. G., and Struhl, K., Eds. 1987. Protocols in Molecular Biology. John Wiley, New York.

2. Bergelson, J., Kreitman, M., Stahl, E., and Tian, D. 2001. Evolutionary dynamics of plant $R$-genes. Science 292:2281-2285.

3. Bryan, G. T., Wu, K., Farrall, L., Jia, Y., Hershey, H. P., McAdams, S., Tarchini, R., Donaldson, G., Faulk, K., and Valent, B. 2000. A single amino acid difference distinguishes resistant and susceptible alleles of the rice blast resistance gene Pi-ta. Plant Cell 12:2033-2045.

4. Caicedo, A., Schaal, B. A., and Kunkel, B. N. 1999. Diversity and molecular evolution of the RPS2 resistance gene in Arabidopsis thaliana. Proc. Natl. Acad. Sci. 96:302-306.

5. Chao, C. T., Moldenhauer, K. A. K., and Ellingboe, A. H. 1999. Genetic analysis of resistance/susceptibility in individual $F_{3}$ families of rice against strains of Magnaporthe grisea containing different genes for avirulence. Euphytica 109:183-190.

6. Correa-Victoria, F. J., Zeigler, R. S., and Levy, M. 1994. Virulence characteristics of genetic families of Pyricularia grisea in Colombia. Pages 211-229 in: Rice Blast Disease. R. S. Ziegler, S. A. Leong, and P. S. Teng, Eds. CAB International, Wallingford, U.K.

7. Feinberg, A. P., and Fogelstein, B. 1983. A technique for radiolabeling DNA fragments to high specific activity. Anal. Biochem. 132:6-13.

8. Grant, M. R., Godiard, L., Straube, E., Ashfield, T., Lewald, J., Sattler, A., Innes, R. W., and Dangl, J. L. 1995. Structure of the Arabidopsis RPM1 gene enabling dual specificity disease resistance. Science 269: 843-846.

9. Hittalmani, S., Parco, A., Mew, T. W., Zeigler, R. S., and Huang, N. 2000. Fine mapping and DNA marker-assisted pyramiding of the three major genes for blast resistance in rice. Theor. Appl. Genet. 100:11211128.

10. Hulbert, S. H., Webb, C. A., Smith, S. M., and Sun, Q. 2001. Resistance gene complexes: Evolution and utilization. Annu. Rev. Phytopathol. 39: 285-312.
11. Jia, Y., Loh, Y.-T., Zhou, J., and Martin, G. B. 1997. Alleles of Pto and Fen occur in bacterial speck-susceptible and fenthion-insensitive tomato lines and encode active protein kinases. Plant Cell 9:61-73.

12. Jia, Y., McAdams, S. A., Bryan, G. T., Hershey, H. P., and Valent, B. 2000. Direct interaction of resistance gene and avirulence gene products confers rice blast resistance. EMBO (Eur. Mol. Biol. Org.) J. 19:4004-4014.

13. Kiyosawa, S. 1976. Pathogenic variations of Pyricularia oryzae and their use in genetic and breeding studies. SABRAO J. 8:53-67.

14. Kiyosawa, S. 1984. Establishment of differential cultivars for pathogenicity test of rice blast fungus. Rice Genet. Newsl. 1:95-97.

15. Kozak, M. 1989. The scanning model for translation: An update. J. Cell. Biol. 108:229-241.

16. Levy, M., Correa-Victoria, F. J., Zeigler, R. S., Xu, S., and Hamer, J. E. 1993. Genetic diversity of the rice blast fungus in a disease nursery in Colombia. Phytopathology 83:1427-1433.

17. Mackill, D. J., and Bonman, J. M. 1992. Inheritance of blast resistance in near-isogenic lines of rice. Phytopathology 82:746-749.

18. Mackill, D. J., Bonman, J. M., McCouch, S. R., Guiderdoni, E., Nottenghem, J. L., and Tanksley, S. D. 1996. Molecular mapping of genes for resistance to rice blast (Pyricularia grisea Sacc.). Theor. Appl. Genet. 93:859-863.

19. Moldenhauer, K. A. K., Lee, F. N., Norman, R. J., Helms, R. S., Well, R. H., Dilday, R. H., Rohman, P. C., and Marchetti, M. A. 1990. Registration of 'Katy' rice. Crop Sci. 30:747-748.

20. Moldenhauer, K. A. K., Bastawisi, A. O., and Lee, F. N. 1992. Inheritance of resistance in rice to races IB-49 and IC-17 of Pyricularia grisea rice blast. Crop Sci. 32:584-588.

21. Moldenhauer, K. A. K., Gravois, K. A., Lee, F. N., Norman, R. J., Bernhardt, J. L., Well, B. R., Dilday, R. H., Blocker, M. M., Rohman, P. C., and McMinn, T. A. 1998. Registration of 'Drew' rice. Crop Sci. 38:896-897.

22. Montenegro-Chamorro, M. V. 1997. Alleleic diversity of an avirulence gene in Colombian field isolates of the rice blast fungus. M.S. thesis. Purdue University, West Lafayette, IN.

23. Orbach, M. J., Farrall, L., Sweigard, J. A., Chumley, F. G., and Valent, B. 2000. A telomeric avirulence gene determines efficacy for the rice blast resistance gene Pi-ta. Plant Cell 12:2019-2023.

24. Rybka, K., Miyamoto, M., Ando, I., Saito, A., and Kawasaki, S. 1997. High resolution mapping of the indica-derived rice blast resistance genes. II. Pi-ta ${ }^{2}$ and Pi-ta and a consideration of their origin. Mol. PlantMicrobe Interact. 10:517-524.

25. Silué, D., Nottenghem, J. L., and Tharreau, D. 1992. Evidence for a gene-for-gene relationship in the Oryza sativa-Magnaporthe grisea pathosystem. Phytopathology 82:577-580.

26. Stahl, E., Dwyer, G., Mauricio, R., Kreitman, M., and Bergelson, J. 1999. Dynamics of disease resistance polymorphism at the Rpml locus of Arabidopsis. Nature 400:667-671.

27. Tian, D., Araki, H., Stahl, E., Bergelson, J., and Kreitman, M. 2002. Signature of balancing selection in Arabidopsis. Proc. Natl. Acad. Sci. U.S.A. 99:11525-11530.

28. Tian, D., Traw, M. B., Chen, J. Q., Kreitman, M., and Bergelson, J. 2003. Fitness costs of $R$-gene-mediated resistance in Arabidopsis thaliana. Nature 423:74-77.

29. Valent, B. 1997. The rice blast fungus, Magnaporthe grisea. Plant relationships. Pages 37-54 in: The Mycota V. Part B. G. C. Carroll and P. Tudzynoski, Eds. Springer-Verlag, Berlin, Germany.

30. Wang, Z.-X., Yano, M., Yamanouchi, U., Iwamoto, M., Monna, L., Hayasaka, H., Katayose, Y., and Sasaki, T. 1999. The Pib gene for rice blast resistance belongs to the nucleotide binding and leucine-rich repeat class of plant disease resistance genes. Plant J. 19:55-64.

31. Yu, J., Hu, S., Wang, J., Wong, G. K., Li, S., Liu, B., Deng, Y., Dai, L., Zhou, Y., Zhang, X., et al. 2002. A draft sequence of the rice genome (Oryza sativa L. ssp. indica). Science 296:79-92.

32. Yu, Z. H., Mackill, D. J., Bonman, J. M., McCouch, S. R., Guiderdoni, E., Notteghem, J. L., and Tanksley, S. D. 1996. Molecular mapping of genes for resistance to rice blast (Pyricularia grisea Sacc.). Theor. Appl. Genet. 93:859-863.

33. Zeigler, R. S., Tohme, J., Nelson, R., Levy, M., and Correa-Victoria, F. J. 1994. Lineage exclusion: A proposal for linking blast population analysis to resistance breeding. Pages 267-292 in: Rice Blast Disease. R. S. Zeigler, S. A. Leong, and P. S. Teng, Eds. CAB International, Wallingford, U.K. 